\title{
EXPERIÊNCIAS PERCEBIDAS DE DISCRIMINAÇ̃̃O E SAÚDE MENTAL: RESULTADOS EM ESTUDANTES UNIVERSITÁRIOS BRASILEIROS
}

\author{
PERCEIVED EXPERIENCES OF DISCRIMINATION AND MENTAL HEALTH: \\ OUTCOMES IN BRAZILIAN UNDERGRADUATES
}

\author{
Amilton dos Santos Júnior ${ }^{1}$ \\ Luiz Lippi Rachkorsky ${ }^{2}$ \\ Pablo Ronzoni ${ }^{3}$ \\ Nisha Dogra ${ }^{4}$ \\ Paulo Dalgalarrondo ${ }^{5}$
}

\section{RESUMO}

Há cada vez mais evidências de associações abordando a percepção de discriminação e seu impacto prejudicial como perpetuador de disparidades de saúde física e mental. O objetivo é usar métodos descritivos para contribuir em uma melhor compreensão das relações entre diferentes categorias de percepção de discriminação e grupos específicos de sintomas mentais e comportamentais. Este é um estudo transversal, realizado na Universidade de Campinas (UNICAMP), uma instituição pública brasileira, com a coleta de diversas variáveis independentes sociodemográficas e as variáveis dependentes foram os resultados da saúde mental, cuja presença foi avaliada através da utilização de instrumentos quantitativos. Os dados pareados foram digitados e analisados usando SPSS, versão 16.0 (SPSS, Inc., Chicago, IL, EUA). Foram realizadas análises de regressão logística, com ambos os modelos uni e multivariada para estudo da associação das variáveis experiências percebidas de discriminação foram frequentemente relatadas $(69,4 \%)$. Graduandos negros/pardos relataram mais discriminação com base na cor da pele e desempenho acadêmico. A partir dos resultados da avaliação da saúde mental, as queixas de depressão, ansiedade e abuso de substância eram muito comuns. Queixas psicológicas, uso de substâncias psicoativas e consequentes comportamentos

\footnotetext{
${ }^{1}$ Professor do Departamento de Psicologia Médica e Psiquiatria da Faculdade de Ciências Médicas da UNICAMP. Telefone: 5519 35217206. E-mail: amilton@fcm.unicamp.br.

${ }^{2}$ Médico Residente do Departamento de Psicologia Médica e Psiquiatria da Faculdade de Ciências Médicas da UNICAMP. E-mail: luizlr@gmail.com.

${ }^{3}$ Consultor em Psiquiatria de Infância e Adolescência - National Health Service (NHS) Trust, Reino Unido. E-mail: pablo.ronzoni@nhs.net.

${ }^{4}$ Professora do Greenwood Institute of Child Health, University of Leicester, Leicester, UK, Reino Unido. E-mail: nd13@leicester.ac.uk.

${ }^{5}$ Professor Titular do Departamento de Psicologia Médica e Psiquiatria da Faculdade de Ciências Médicas da UNICAMP. E-mail: pdalga@fcm.unicamp.br.
} 
de risco foram frequentes entre o grupo de estudantes de graduação brasileiros investigados neste estudo, assim como percepções de diferentes categorias de experiências discriminatórias. Estudos futuros deverão explorar o impacto longitudinal de cada categoria de discriminação percebida e associações causais com diagnósticos psiquiátricos específicos, consequências adversas pessoais e sociais e a busca de ajuda ou tratamento.

PALAVRAS-CHAVE: Cor de pele. Discriminação. Ensino Superior. Preconceito. Saúde. Mental

\begin{abstract}
There is increasing evidence of associations addressing the perception of discrimination and its adverse impact as perpetrator of physical and mental health disparities. The goal is to use descriptive methods to contribute to a better understanding of the relationships between different categories of perception of discrimination and specific groups of mental and behavioral symptoms. This cross-sectional study, conducted at the University of Campinas (UNICAMP), a Brazilian public institution, with the collection of several sociodemographic independent variables and the mental health dependent variables were assessed by using quantitative tools. Paired data were entered and analyzed using SPSS version 16.0 (SPSS, Inc., Chicago, IL, USA). Logistic regression analyzes were performed with both uni- and multivariate models to study the association of variables. Perceived discrimination experiences were frequently reported (69.4\%). Black/brown undergrads reported more discrimination based on skin color and academic achievement. From the evaluation results of mental health complaints of depression, anxiety and substance abuse were common. Psychological complaints, substance abuse and consequent risk behaviors were common among the group of Brazilian graduate students investigated in this study as well as perceptions of different types of discrimination experiments. Future studies should explore the longitudinal impact of each category of perceived discrimination and causal associations with specific psychiatric diagnoses, personal and social adverse consequences and seeking help or treatment.
\end{abstract}

KEYWORDS: Skin color. Discrimination. Higher Education. Racism. Mental Health.

\title{
INTRODUÇÃO
}

A exposição a sérios estressores psicológicos relacionados com o status de identidade apresenta desafios específicos para a Psiquiatria. Experiências de percepção de discriminação são estressores importantes, o que pode perpetuar os problemas sociais, precipitar sintomas psicológicos ou complicar transtornos psiquiátricos préexistentes, segundo Kelly (2007). Bhugra (2001) destaca que as práticas discriminatórias podem operar tanto em nível individual como institucional, levando a problemas como os estereótipos, rejeição, preconceito, a desvalorização de uma cultura particular, ameaças e até mesmo ataques.

Serv. Soc. \& Saúde, Campinas, SP v. 14, n. 2 (20), p. 273-298, jul./dez. 2015 ISSN 1676-6806 
Há cada vez mais evidências de associações abordando a percepção de discriminação e seu impacto prejudicial como perpetuador de disparidades de saúde física e mental. Embora os dados sejam tipicamente transversais, Noh (2007) e Ellis (2008) ressaltam que o elo teórico é que as experiências discriminatórias servem como um estressor e precipitam vários problemas de saúde mental. Embora seja difícil distinguir objetivamente entre a discriminação percebida e a real, Landrine (2006) e Hwang (2008) destacam que a mera percepção de ser discriminado é suficiente para diminuir o estado de saúde e saúde mental das pessoas que o experimentam.

Como uma questão preocupante, Oliveira (2008) percebeu que há uma crescente consciência em relação à saúde mental dos estudantes universitários, que enfrentam não somente os desafios de completar o Ensino Superior, mas também muitos problemas de desenvolvimento que acompanham o final da adolescência e início da vida adulta. Estes indivíduos, como estudado por Prelow (2006); Lahiri (2007) e Lo (2009), são particularmente suscetíveis a experimentar eventos discriminatórios e as suas consequências que, se não forem devidamente manejadas, podem persistir até a idade adulta e causar graves problemas acadêmicos e interpessoais. Grunbaum (2004); Viner (2006) e The International Association of Counseling Services (2009) observaram que quadros depressivos e de ansiedade, angústia e perda de autoestima são comuns durante este período do desenvolvimento humano. Observa-se também, segundo os estudos de Somers (2004), Barret (2006), Hingson (2005) e Blanco (2008), que o álcool e o uso de múltiplas substâncias são frequentes entre esses indivíduos, sendo muitas vezes associados com abuso, dependência e comportamentos de risco.

A ampla gama de efeitos na saúde mental relacionados com o impacto negativo da discriminação lança luz sobre as implicações de longo alcance desta questão social. Alguns podem argumentar que uma amostra de uma universidade pode reduzir as diferenças de grupo em experiências discriminatórias, pois pode haver semelhanças contextuais e seleção de indivíduos com melhor resiliência e mais alto funcionamento cognitivo. McCormack (1996), porém, traz evidências sugerindo que a discriminação nos campus tem aumentado ao longo dos anos.

Sem desconsiderar possíveis vieses relacionados à cor da pele e distribuição socioeconômica dos estudantes inquiridos, este estudo não pretende inferir relações causais. O objetivo é usar métodos descritivos para contribuir em uma melhor 
compreensão das relações entre diferentes categorias de percepção de discriminação e grupos específicos de sintomas mentais e comportamentais, em uma amostra de estudantes universitários brasileiros.

\section{MÉTODOS}

\section{AMOSTRAGEM}

O estudo foi realizado na Universidade Estadual de Campinas (UNICAMP), uma instituição pública brasileira, fundada em outubro de 1966. Apesar de ser uma universidade jovem, a UNICAMP já desenvolveu uma forte tradição no ensino, pesquisa e serviço à sociedade. Seu complexo é constituído de três campus e uma série de unidades de apoio dentro de um universo de cerca de 50.000 pessoas. A Universidade tem cerca de 35.000 alunos, dos quais cerca de 19.000 são alunos de graduação, segundo informações do site da própria universidade em 2016. A admissão em cursos de graduação é permitida para indivíduos que tenham concluído o Ensino Médio e que tenham sido aprovados em testes competitivos anuais. Estatísticas analisadas de Oliveira (2008) relatam que $80,8 \%$ dos alunos de graduação admitidos na UNICAMP em 2004 eram adultos jovens, com idades entre 17 e 20 anos.

Em dezembro de 2004 foi realizado um estudo-piloto, que incluiu 60 alunos de graduação dos cursos de Medicina e Fonoaudiologia, a fim de avaliar a aceitação e inteligibilidade do questionário e para definir o tamanho da amostra do estudo principal.

A amostra total coberta pelo banco de dados foi planejada de acordo com cálculos para o tamanho da amostra, após o estudo-piloto que determinou uma prevalência de cerca de $30 \%$ para transtornos mentais com o nível de significância de $1 \%($ alfa $=0,01)$ e erro amostral de $d=0,035$. O tamanho mínimo da amostra que devia ser pesquisada a partir do total de 14.640 alunos regularmente matriculados na Universidade de Campinas em 2005 foi estimado em 1.138 indivíduos. A coleta de dados ocorreu entre outubro de 2005 e novembro de 2006. Um total de 1.306 questionários foram efetivamente recolhidos. Neves (2007) ressalta que os resultados Serv. Soc. \& Saúde, Campinas, SP v. 14, n. 2 (20), p. 273-298, jul./dez. 2015 ISSN 1676-6806 
gerais dos transtornos mentais auto referidos tomados a partir desta amostra são apresentados em outros lugares. À medida que a sociedade brasileira é composta principalmente de mistos de etnias/raças, censos oficiais do Instituto Brasileiro de Geografia e Estatística têm usado, desde 1872, o critério auto descrito "cor da pele" em seus métodos, segundo IBGE (2000). De acordo com este sistema de classificação, que não reflete necessariamente uma origem étnica compartilhada, cidadãos brasileiros são auto identificados em três principais grupos de cor de pele: "brancos" (caucasianos), "negros" (afrodescendentes), e "pardos" (misto dos dois acima). Este estudo usou essa classificação oficial brasileira e somente os estudantes que se auto identificaram como de um destes três grupos foram considerados na análise. Portanto, 1.174 alunos foram incluídos, o que constitui $89,8 \%$ da amostra original.

Os critérios de inclusão para os participantes foram: ser estudante universitário, de ambos os sexos, regularmente matriculado em algum curso da UNICAMP (Estado de São Paulo, Brasil) e que tivesse lido, concordado e assinado o termo de consentimento livre e esclarecido. Os alunos do primeiro semestre de seus cursos não foram inquiridos devido a breve exposição ao ambiente acadêmico da Universidade. Alunos especiais (não regularmente matriculados) e aqueles que eram estrangeiros, não fluente em português, também foram excluídos. A seleção da amostra foi baseada em grupos de conglomerados proporcionais de alunos de cada curso. Foi realizada uma seleção aleatória de aulas e programas que participam do estudo, com base na representatividade de cada área do conhecimento. No entanto, como algumas aulas podem ter muitos alunos ausentes e isso poderia resultar em um viés de amostragem importante, apenas as classes com pelo menos 60\% dos alunos regularmente matriculados em sala de aula no momento do pedido foram considerados para o levantamento. Houve 36 cursos contatados, mas apenas em 17 foi possível a coleta de dados, porque muitos coordenadores e professores expressaram preocupação a respeito do tempo de aula perdida. Os cursos participantes foram: Estudos Literários, Pedagogia, Música, Fonoaudiologia, Medicina, Estatística, Física, Arquitetura, Engenharia Civil, Engenharia Agrícola, Engenharia de Alimentos, Engenharia de Computação, Engenharia Química, Informática, Tecnologia de Saneamento Ambiental, Tecnologia da Construção Civil e Tecnologia de Telecomunicações. 
A fim de evitar possíveis más interpretações devido a fatores de confusão, relacionados com a distribuição racial e socioeconômica da população estudantil, a construção da amostra final foi refinada por um processo de emparelhamento estratificado. Isso ocorreu devido a preocupação de que indivíduos de cor da pele negra e parda pudessem estar sub-representados em configurações e posições de poder da universidade, como tem sido observado por Heringer (2002) e Chor (2005), uma vez que estes são mais associados a indicadores sociais e de saúde desfavoráveis. Inicialmente, os indivíduos foram separados de acordo com sua cor da pele auto referida. Posteriormente, foram pareados de acordo com as características socioeconômicas similares. Além disso, para cada estudante autodeclarado negro/pardo, foi escolhido outro que se auto identificou como branco e que compartilhava similaridades nas seguintes características: intervalo de idade; período durante o qual o estudante frequentou aulas na Universidade e se o aluno estava ou não trabalhando. Após esta etapa, a distribuição da amostra para análise foi finalizada com 346 indivíduos, provenientes de dois grupos: um com todos os 173 alunos auto referidos como negros ou pardos (39 negros e 144 pardos), emparelhados com outro grupo, com 173 estudantes brancos, escolhidos dentre os 1.001 indivíduos que se identificaram como pertencentes a este grupo de cor da pele. Estudantes negros e pardos foram considerados em conjunto em um grupo puro porque o número de estudantes negros era muito baixo e também porque há muitos recursos econômicos e políticos compartilhados por eles na sociedade brasileira, bem como relatos de experiências de percepção de discriminação como exemplificado por Carey (2007). Outro estudo, com resultados a partir deste mesmo banco de dados em relação às questões específicas de cor da pele e diferenças entre estudantes universitários negros e pardos, está em andamento.

\section{CONCEPÇÃO E INSTRUMENTOS}

Este é um estudo transversal. As variáveis independentes do questionário para esta pesquisa foram: sexo, intervalo de idade (menos de 21;21-25; mais de 25 anos de idade); cor da pele (branco ou negro/pardo); situação socioeconômica (alta, média ou baixa); período em que o aluno teve aulas na universidade (durante o dia ou à noite); se Serv. Soc. \& Saúde, Campinas, SP v. 14, n. 2 (20), p. 273-298, jul./dez. 2015 ISSN 1676-6806 
o estudante também estava trabalhando ou não; e diferentes tipos de experiências percebidas de discriminação pelo menos uma vez na vida. Essas percepções incluíram respostas afirmativas às perguntas de sentimento de discriminação por qualquer motivo não especificado e/ou devido a uma das seguintes categorias: aparência física, status socioeconômico, desempenho acadêmico, posições políticas, roupas e adereços corporais, religião, cor da pele e orientação sexual. Como a distribuição dos alunos de acordo com sua área de curso neste questionário não reflete a distribuição real da Universidade (muitos cursos não participaram devido à recusa do coordenador), esta variável não foi considerada para a análise multivariada. As variáveis dependentes foram os resultados da saúde mental, cuja presença foi avaliada através da utilização dos seguintes instrumentos quantitativos:

\section{A) M.I.N.I.}

O M.I.N.I. (Mini Internacional Neuropsychiatric Interview), que é uma breve e padronizada entrevista diagnóstica compatível com os critérios do DSM-III-R e CID-10 25 , foi utilizado para avaliar possíveis transtornos mentais. Os resultados de fiabilidade e validade do instrumento, realizado por Amorim (2000), são satisfatórios para o desenvolvimento da versão brasileira e tem havido uma combinação de procedimentos epidemiológicos e antropológicos a fim de otimizar a sensibilidade cultural do instrumento.

No presente estudo, o M.I.N.I. foi usado como um inventário de auto relato de queixas subjetivas descritas pelo aluno. Os sintomas que sugerem os seguintes transtornos psiquiátricos foram considerados: episódio depressivo maior; transtorno distímico; transtorno do pânico; agorafobia; fobia social; transtorno obsessivo compulsivo; bulimia nervosa; transtorno de ansiedade generalizada. Também foram considerados a insônia por pelo menos duas semanas durante os últimos 12 meses e pensamentos suicidas pelo menos uma vez na vida. Para a análise estatística, a variável "qualquer categoria afirmativa avaliada pelo M.I.N.I." foi criada, e esta variável foi considerada positiva quando incluía uma resposta afirmativa a, pelo menos, uma das categorias anteriores, exceto insônia e pensamentos suicidas. Outros agrupamentos sugestivos de efeitos na saúde mental possivelmente avaliadas pelo M.I.N.I., tais como traços antissociais ou experiências de estresse psicóticos e pós-traumáticas, não foram 
incluídos no estudo devido à baixa prevalência presumida destas condições nesta amostra.

B) AUDIT

O AUDIT (Alcohol Use Disorder Identification Test) é um instrumento de rastreamento desenvolvido pela OMS em 1980 e validado no Brasil. Esta escala tem como objetivo identificar as pessoas em risco para o consumo, abuso e dependência de álcool. A pontuação varia de 0 a 40 , e uma pontuação de oito ou mais indica a necessidade de um diagnóstico mais específico, segundo Babor (1992) e Lima (2005). Assim, este corte foi usado para considerar o rastreio da variável "uso mais frequente de álcool" como positivo.

\section{C) IDENTIFICAÇÃO DO USO DE SUBSTÂNCIAS PSICOATIVAS ILÍCITAS}

Um questionário baseado no Centro Brasileiro de Informações sobre Drogas Psicotrópicas - CEBRID - foi o método utilizado para identificar o uso de substâncias psicoativas ilícitas, incluindo as seguintes: maconha, cocaína em pó, crack, anfetaminas, alucinógenos, ecstasy, solventes voláteis, esteroides anabolizantes ou outras substâncias utilizadas com a finalidade de "ficar chapado". O questionário não teve o objetivo de fazer os diagnósticos clínicos de abuso ou dependência. Para cada substância considerada, havia as seguintes respostas possíveis: (1) Nunca utilizada; (2) usada pelo menos uma vez na vida; (3) usada pelo menos uma vez nos últimos 12 meses; (4) utilizada durante os últimos 30 dias. $\mathrm{O}$ questionário adaptado foi considerado positivo quando o estudante relatou "sim" à pergunta de ter já utilizado maconha pelo menos uma vez durante os últimos 30 dias ou qualquer outra substância psicoativas ilícitas pelo menos uma vez na vida.

D) Comportamentos de risco após beber ou usar outra substância psicoativa

Esta variável foi construída de acordo com respostas positivas para pelo menos uma das seguintes questões: (1) "Você já dirigiu um veículo depois de beber uma bebida alcoólica ou após o uso de alguma outra substância psicoativa?"; (2) "Você já Serv. Soc. \& Saúde, Campinas, SP v. 14, n. 2 (20), p. 273-298, jul./dez. 2015 ISSN 1676-6806 
teve relações sexuais com uma pessoa desconhecida, depois de beber uma bebida alcoólica ou após o uso de alguma outra substância psicoativa?”.

\section{CONSIDERAÇÕES ÉTICAS}

Para a coleta de dados, a aprovação ética do Comitê de Ética em Pesquisa da Faculdade de Ciências Médicas da Universidade Estadual de Campinas (CEP / FCM / UNICAMP) foi obtida em agosto de 2004 (N. $\left.{ }^{\circ} 331\right)$.

\section{ANÁLISE}

Os dados pareados foram digitados e analisados usando SPSS, versão 16.0 (SPSS, Inc., Chicago, IL, EUA). Tabelas descritivas foram criadas para mostrar as características sócio demográficas da amostra, as taxas de experiências percebidas de discriminação e a prevalência das consequências avaliadas na saúde mental dos indivíduos. Análise bivariada simples foi utilizada para comparar universitários negros e pardos com brancos e testes qui-quadrado avaliou a importância dos dados. P-valores menores que 0,05 foram considerados estatisticamente significativos.

Em relação a efeitos na saúde mental possivelmente associados com as características sócio demográficas e experiências percebidas de discriminação, análises de regressão logística, com ambos os modelos uni e multivariada, foram realizadas no estudo da associação das variáveis. Das variáveis dependentes, apenas aquelas com uma prevalência de mais de 15\% na amostra final emparelhada foram consideradas para os modelos, com exceção da categoria "pensamentos suicidas pelo menos uma vez na vida", por causa de seu impacto prejudicial em relação a todos os efeitos na saúde mental e porque, segundo Abdel-Khalek (2002) qualquer tipo de distúrbio psiquiátrico pode aumentar o risco de suicídio. Análises de regressão logística univariadas foram inicialmente realizadas a fim de ajustar o modelo para as variáveis dependentes, relativamente a cada uma delas. Este procedimento foi seguido por critérios graduais para selecionar as categorias que melhor explicaram as variáveis de interesse, obtendose assim modelos para a regressão logística multivariada, que são mostrados na seção de Serv. Soc. \& Saúde, Campinas, SP v.15, n. 2 (22), p. 273-298, jul./dez. 2016 ISSN 1676-6806 
resultados abaixo. O nível de significância foi de $5 \%(\mathrm{p} \leq 0,05)$. O Sistema SAS for Windows (Statistical Analysis System), versão 9.1.3 (SAS Institute Inc, 2002-2003, Cary, NC, EUA) foi utilizado para obter a regressão logística e análise multivariada.

\section{RESULTADOS}

Intencionalmente, de acordo com os critérios de estratificação da composição da amostra final, não houve diferenças estatisticamente significativas entre os grupos de cor de pele nas seguintes características: intervalos de idade, status socioeconômico, se o aluno também trabalha e hora do dia durante o qual os indivíduos assistiram às aulas na Universidade.

Como grupo de estudantes brancos foi organizado a partir das características socioeconômicas e demográficas dos estudantes negros e pardos, ao analisar ambos os grupos em conjunto, a maioria dos estudantes tinha menos de 26 anos $(73,4 \%)$ e provinha de famílias de nível socioeconômico baixo ou médio (76,6\%). Mais da metade também trabalhava $(55,5 \%)$ e cursava o período diurno $(57,8 \%)$. A distribuição por sexo foi quase idêntica entre os dois grupos de cor de pele, com um predomínio do sexo feminino $(58,1 \%)$. Em relação a suas áreas do curso, os alunos de graduação foram distribuídos em: Ciências Exatas e Áreas Tecnológicas (46,2\%); Ciências Humanas e Artes (35,8\%) e profissionais da Saúde (17,9\%).

Experiências percebidas de discriminação foram frequentemente relatadas $(69,4 \%)$. As categorias mais citadas foram à discriminação com base na aparência física e no nível socioeconômico. Graduandos negros/pardos relataram mais discriminação, estatisticamente, do que os brancos nas categorias com base na cor da pele e desempenho acadêmico. As frequências descritivas que mostram as taxas de experiências de discriminação percebida relatadas são mostradas na Tabela 1. 
Tabela 1- Frequências descritivas da composição da amostra final $(n=346)$, incluindo relatos de experiências percebidas de discriminação

\begin{tabular}{|c|c|c|c|c|}
\hline $\begin{array}{l}\text { Categorias de } \\
\text { discriminação }\end{array}$ & $\begin{array}{c}\text { Negros e } \\
\text { Pardos } \\
\text { n (\%) }\end{array}$ & $\begin{array}{c}\text { Brancos } \\
\text { n (\%) }\end{array}$ & P-valor & $\begin{array}{l}\text { Total } \\
\text { n (\%) }\end{array}$ \\
\hline \multicolumn{5}{|c|}{ Qualquer relato de percepção de experiência discriminatória } \\
\hline Sim & $134(77,5 \%)$ & $106(61,3 \%)$ & 0,001 & $240(69,4 \%)$ \\
\hline Não & $39(22,5 \%)$ & $67(38,7 \%)$ & & $106(30,6)$ \\
\hline \multicolumn{5}{|c|}{ Discriminação contra aparência física } \\
\hline Sim & $61(35,3 \%)$ & $47(27,2 \%)$ & $0,104^{*}$ & $108(31,2 \%)$ \\
\hline Não & $112(64,7 \%)$ & $126(72,8 \%)$ & & $238(68,8 \%)$ \\
\hline \multicolumn{5}{|c|}{ Discriminação contra status social } \\
\hline Sim & $53(30,6 \%)$ & $41(23,7 \%)$ & $0,147^{*}$ & $94(27.2 \%)$ \\
\hline Não & $120(69,4 \%)$ & $132(76,3 \%)$ & & $252(72.8 \%)$ \\
\hline \multicolumn{5}{|c|}{ Discriminação contra roupas e adereços corporais } \\
\hline Sim & $31(17,9 \%)$ & $24(13,9 \%)$ & $0,303^{*}$ & $55(15,9 \%)$ \\
\hline Não & $142(82,1 \%)$ & $149(86,1 \%)$ & & $291(84,1 \%)$ \\
\hline \multicolumn{5}{|c|}{ Discriminação contra religião } \\
\hline Sim & $21(12,1 \%)$ & $21(12,1 \%)$ & $1,000^{*}$ & $42(12,1 \%)$ \\
\hline Não & $152(87,9 \%)$ & $152(87,9 \%)$ & & $304(87,9 \%)$ \\
\hline \multicolumn{5}{|c|}{ Discriminação contra cor da pele } \\
\hline Sim & $27(15,6 \%)$ & $4(2,3 \%)$ & $<0,001$ & $31(9,0 \%)$ \\
\hline Não & $146(84,4 \%)$ & $169(97,7 \%)$ & & $315(91,0 \%)$ \\
\hline \multicolumn{5}{|c|}{ Discriminação contra desempenho acadêmico } \\
\hline Sim & $20(11,6 \%)$ & $9(5,2 \%)$ & 0,033 & $29(8,4 \%)$ \\
\hline Não & $153(88,4 \%)$ & $164(94,8 \%)$ & & $317(91,6 \%)$ \\
\hline \multicolumn{5}{|c|}{ Discriminação contra posições políticas } \\
\hline Sim & $16(9,2 \%)$ & $10(5,8 \%)$ & $0,221^{*}$ & $26(7,5 \%)$ \\
\hline Não & $157(90,8 \%)$ & $163(94,2 \%)$ & & $320(92,5 \%)$ \\
\hline \multicolumn{5}{|c|}{ Discriminação contra orientação sexual } \\
\hline Sim & $8(4,7 \%)$ & $7(4,1 \%)$ & $0,783^{*}$ & $15(4,3 \%)$ \\
\hline Não & $161(95,3 \%)$ & $163(95,9 \%)$ & & $324(93,6 \%)$ \\
\hline Sem resposta & & & & $7(2,0 \%)$ \\
\hline
\end{tabular}

Fonte: Pesquisa de Campo (DOS SANTOS-JÚNIOR, 2011)

*Sem significância estatística $(\mathrm{p}>0,05)$

A partir dos resultados da avaliação da saúde mental, as queixas de depressão e ansiedade foram muito comuns, bem como os comportamentos relacionados ao uso de substâncias psicoativas, incluindo o álcool. A única categoria em que houve diferença entre os grupos de cor de pele foi episódio depressivo maior $(\mathrm{p}=0,025)$, com maior prevalência de relatos entre negros e pardos $(26,2 \%)$ do que entre os brancos $(16,3 \%)$, 
Os dados de resultados psicológicos e comportamentais de cada grupo e de toda a amostra estão apresentados na Tabela 2.

Tabela 2 - Prevalência de efeitos na Saúde Mental

\begin{tabular}{|c|c|c|c|c|c|}
\hline & & $\begin{array}{l}\text { Negros e } \\
\text { Pardos } \\
\text { n=173 (\%) }\end{array}$ & $\begin{array}{l}\text { Brancos } \\
\mathrm{n}=1001(\%) \\
\end{array}$ & P-valor & $\begin{array}{l}\text { Total } \\
\text { n (\%) } \\
\end{array}$ \\
\hline $\begin{array}{l}\text { Qualquer } \\
\text { afirmação } \\
\text { positiva nas } \\
\text { categorias } \\
\text { avaliadas no } \\
\text { M.I.N.I. } \\
\end{array}$ & $\begin{array}{l}\text { Sim } \\
\text { Não } \\
\text { Sem resposta }\end{array}$ & $\begin{array}{l}108(62,4 \%) \\
65(37,6 \%)\end{array}$ & $\begin{array}{l}990(99,3 \%) \\
5(0,5 \%)\end{array}$ & $0,481^{*}$ & $\begin{array}{l}209(60,4 \%) \\
136(39,3 \%) \\
1(0,3 \%) \\
\end{array}$ \\
\hline $\begin{array}{l}\text { Queixas } \\
\text { depressivas } \\
\text { episódicas e } \\
\text { sérias } \\
\text { atualmente }\end{array}$ & $\begin{array}{l}\text { Sim } \\
\text { Não } \\
\text { Sem resposta }\end{array}$ & $\begin{array}{l}45(26,2 \%) \\
127(73,8 \%)\end{array}$ & $\begin{array}{l}28(16,3 \%) \\
144(83,7 \%)\end{array}$ & 0,025 & $\begin{array}{l}73(21,1 \%) \\
271(78,3 \%) \\
2(0,6 \%)\end{array}$ \\
\hline $\begin{array}{l}\text { Queixas } \\
\text { depressivas } \\
\text { leves, porém } \\
\text { persistentes }\end{array}$ & $\begin{array}{l}\text { Sim } \\
\text { Não } \\
\text { Sem resposta }\end{array}$ & $\begin{array}{l}44(25,6 \%) \\
128(74,4 \%)\end{array}$ & $\begin{array}{l}32(18,6 \%) \\
140(81,4 \%)\end{array}$ & $0,119^{*}$ & $\begin{array}{l}76(22,0 \%) \\
268(77,5 \%) \\
2(0,6 \%) \\
\end{array}$ \\
\hline $\begin{array}{l}\text { Quadros } \\
\text { ansiosos } \\
\text { paroxísticos } \\
\text { atuais }\end{array}$ & $\begin{array}{l}\text { Sim } \\
\text { Não } \\
\text { Sem resposta }\end{array}$ & $\begin{array}{l}27(15,8 \%) \\
144(84,2 \%)\end{array}$ & $\begin{array}{l}30(17,5 \%) \\
141(82,5 \%)\end{array}$ & $0,663^{*}$ & $\begin{array}{l}57(16,5 \%) \\
285(82,4 \%) \\
4(1,2 \%)\end{array}$ \\
\hline $\begin{array}{l}\text { Sintomas } \\
\text { persistentes } \\
\text { de ansiedade, } \\
\text { mais que o } \\
\text { habitual }\end{array}$ & $\begin{array}{l}\text { Sim } \\
\text { Não } \\
\text { Sem resposta }\end{array}$ & $\begin{array}{l}75(44,1 \%) \\
95(55,9 \%)\end{array}$ & $\begin{array}{l}69(40,1 \%) \\
103(59,9 \%)\end{array}$ & $0,454^{\star}$ & $\begin{array}{l}144(41,6 \%) \\
198(57,2 \%) \\
4(1,2 \%) \\
\end{array}$ \\
\hline $\begin{array}{l}\text { Insônia por, } \\
\text { pelo menos, } 2 \\
\text { semanas nos } \\
\text { últimos } 12 \\
\text { meses }\end{array}$ & $\begin{array}{l}\text { Sim } \\
\text { Não } \\
\text { Sem resposta }\end{array}$ & $\begin{array}{l}64(38,1 \%) \\
104(61,9 \%)\end{array}$ & $\begin{array}{l}57(33,3, \%) \\
114(66,7 \%)\end{array}$ & $0,360^{*}$ & $\begin{array}{l}121(35 \%) \\
218(63 \%) \\
7(2,0 \%) \\
\end{array}$ \\
\hline $\begin{array}{l}\text { Pensamentos } \\
\text { suicidas pelo } \\
\text { menos uma } \\
\text { vez na vida }\end{array}$ & $\begin{array}{l}\text { Sim } \\
\text { Não } \\
\text { Sem resposta }\end{array}$ & $\begin{array}{l}27(16,1 \%) \\
141(83,9 \%)\end{array}$ & $\begin{array}{l}21(12,3 \%) \\
150(87,7 \%)\end{array}$ & $0,317^{*}$ & $\begin{array}{l}48(13,9 \%) \\
291(84,1 \%) \\
7(2,0 \%)\end{array}$ \\
\hline
\end{tabular}

Serv. Soc. \& Saúde, Campinas, SP v. 14, n. 2 (20), p. 273-298, jul./dez. 2015 ISSN 1676-6806 


\begin{tabular}{|c|c|c|c|c|c|}
\hline Continuação & & $\begin{array}{l}\text { Negros e } \\
\text { Pardos } \\
n=173(\%)\end{array}$ & $\begin{array}{l}\text { Brancos } \\
n=1001(\%)\end{array}$ & P-valor & $\begin{array}{l}\text { Total } \\
\text { n (\%) }\end{array}$ \\
\hline $\begin{array}{l}\text { Uso mais } \\
\text { frequente de } \\
\text { álcool }\end{array}$ & $\begin{array}{l}\text { Sim } \\
\text { Não } \\
\text { Sem resposta }\end{array}$ & $\begin{array}{l}32(18,9 \%) \\
137(81,1 \%)\end{array}$ & $\begin{array}{l}37(21,6 \%) \\
134(78,4 \%)\end{array}$ & $0,536^{*}$ & $\begin{array}{l}69(19,9 \%) \\
271(78,3 \%) \\
6(1,7 \%) \\
\end{array}$ \\
\hline $\begin{array}{l}\text { Uso de } \\
\text { substâncias } \\
\text { psicoativas } \\
\text { ilícitas }\end{array}$ & $\begin{array}{l}\text { Sim } \\
\text { Não } \\
\text { Sem resposta }\end{array}$ & $\begin{array}{l}35(20,8 \%) \\
133(79,2 \%)\end{array}$ & $\begin{array}{l}46(26,9 \%) \\
125(73,1 \%)\end{array}$ & $0,190^{*}$ & $\begin{array}{l}81(23,4 \%) \\
258(74,6 \%) \\
7(2,0 \%)\end{array}$ \\
\hline $\begin{array}{l}\text { Comportame } \\
\text { nto de risco } \\
\text { após uso de } \\
\text { álcool ou } \\
\text { outra } \\
\text { substância } \\
\text { psicoativa }\end{array}$ & $\begin{array}{l}\text { Sim } \\
\text { Não } \\
\text { Sem resposta }\end{array}$ & $\begin{array}{l}30(18,8 \%) \\
130(81,3 \%)\end{array}$ & $\begin{array}{l}38(22,6 \%) \\
130(77,4 \%)\end{array}$ & $0,388^{*}$ & $\begin{array}{l}68(19,7 \%) \\
260(75,1 \%) \\
18(5,2 \%)\end{array}$ \\
\hline
\end{tabular}

Fonte: Pesquisa de Campo (Dos Santos-Júnior, 2011)

*Sem significância estatística $(\mathrm{p}>0,05)$

Devido à sua baixa ocorrência (inferior a 15\%), as seguintes categorias não foram consideradas variáveis dependentes para os modelos de regressão logística linear: agorafobia (13\%); fobia social $(7,8 \%)$; bulimia nervosa $(4,9 \%)$; e transtorno obsessivocompulsivo $(1,4 \%)$,

As análises de regressão logística multivariada mostraram que cada variável dependente, relacionada aos agrupamentos de sintomas, apresentou um padrão específico de razão entre chances (odds ratio - OR) significativa de associação com as variáveis independentes. Os dados sobre variáveis associadas com maior OR para aglomerados de queixas psicológicas avaliadas pelo M.I.N.I. estão apresentados na Tabela 3. 
Tabela 3 - Análises de regressão logística multivariada para diagnósticos positivos autoavaliados no M.I.N.I.

\begin{tabular}{|c|c|c|c|c|}
\hline Variáveis selecionadas* & Categorias & P-Valor & $\mathbf{O R}^{* *}$ & IC 95\% OR \\
\hline \multicolumn{5}{|c|}{ Qualquer categoria positiva avaliada pelo M.I.N.I. } \\
\hline Sexo & $\begin{array}{l}\text { Masculino } \\
\text { (ref.) } \\
\text { Feminino }\end{array}$ & $<0,001$ & $\begin{array}{c}1 \\
2,6\end{array}$ & $1,6-4,2$ \\
\hline Idade & $\begin{array}{c}\leq 20 \text { (ref.) } \\
21-25 \\
\geq 26\end{array}$ & $\begin{array}{c}<0,001 \\
0,007\end{array}$ & $\begin{array}{c}1 \\
2,6 \\
2,5\end{array}$ & $\begin{array}{c}--- \\
1,5-4,4 \\
1,3-4,7\end{array}$ \\
\hline $\begin{array}{l}\text { Discriminação por } \\
\text { desempenho acadêmico }\end{array}$ & $\begin{array}{l}\text { Não (ref.) } \\
\text { Sim }\end{array}$ & 0,036 & $\begin{array}{c}1 \\
3,1\end{array}$ & $1,1-8,8$ \\
\hline $\begin{array}{l}\text { Discriminação contra } \\
\text { roupas/adereços corporais }\end{array}$ & $\begin{array}{l}\text { Não (ref.) } \\
\text { Sim }\end{array}$ & 0,039 & $\begin{array}{c}1 \\
2,1\end{array}$ & $\begin{array}{c}--- \\
1,04-4,4\end{array}$ \\
\hline \multicolumn{5}{|c|}{ Queixas depressivas sérias e episódicas atuais } \\
\hline $\begin{array}{l}\text { Discriminação contra } \\
\text { status social }\end{array}$ & $\begin{array}{l}\text { Não (ref.) } \\
\text { Sim }\end{array}$ & 0,008 & $\begin{array}{c}1 \\
2,2\end{array}$ & $\begin{array}{c}-- \\
1,2-3,9\end{array}$ \\
\hline Idade & $\begin{array}{c}\leq 20 \text { (ref.) } \\
21-25 \\
\geq 26\end{array}$ & $\begin{array}{l}0,039 \\
0,006\end{array}$ & $\begin{array}{c}1 \\
2,2 \\
3\end{array}$ & $\begin{array}{c}--- \\
1,04-4,6 \\
1,4-6,7\end{array}$ \\
\hline $\begin{array}{l}\text { Discriminação contra } \\
\text { aparência física }\end{array}$ & $\begin{array}{l}\text { Não (ref.) } \\
\text { Sim }\end{array}$ & 0,038 & $\begin{array}{c}1 \\
1,8 \\
\end{array}$ & $\begin{array}{c}--- \\
1,04-3,2 \\
\end{array}$ \\
\hline \multicolumn{5}{|c|}{ Queixas depressivas leves mas persistentes } \\
\hline $\begin{array}{l}\text { Discriminação contra } \\
\text { status social }\end{array}$ & $\begin{array}{l}\text { Não (ref.) } \\
\text { Sim }\end{array}$ & 0,021 & $\begin{array}{c}1 \\
2,1\end{array}$ & $\begin{array}{c}--- \\
1,1-3,9\end{array}$ \\
\hline Sexo & $\begin{array}{l}\text { Masculino } \\
\text { (ref.) } \\
\text { Feminino }\end{array}$ & 0,005 & $\begin{array}{c}1 \\
2,5 \\
\end{array}$ & $1,3-4,6$ \\
\hline Idade & $\begin{array}{c}\leq 20 \text { (ref.) } \\
21-25 \\
\geq 26\end{array}$ & $\begin{array}{l}0,010 \\
0,003\end{array}$ & $\begin{array}{c}1 \\
2,7 \\
3,4\end{array}$ & $\begin{array}{c}--- \\
1,2-5,9 \\
1,5-7,6\end{array}$ \\
\hline $\begin{array}{l}\text { Qualquer relato de } \\
\text { experiência de } \\
\text { discriminação }\end{array}$ & $\begin{array}{l}\text { Não (ref.) } \\
\quad \text { Sim }\end{array}$ & 0,038 & $\begin{array}{c}1 \\
2,3\end{array}$ & $1,05-5,2$ \\
\hline \multicolumn{5}{|c|}{ Episódios paroxísticos atuais com sintomas de ansiedade } \\
\hline Idade & $\begin{array}{c}\leq 20 \text { (ref.) } \\
21-25 \\
\geq 26\end{array}$ & $\begin{array}{c}0,198^{* * *} \\
0,003\end{array}$ & $\begin{array}{c}1 \\
1,7 \\
3,5\end{array}$ & $\begin{array}{c}--- \\
0,8-3,8 \\
1,5-7,8\end{array}$ \\
\hline $\begin{array}{l}\text { Discriminação contra } \\
\text { posições políticas }\end{array}$ & $\begin{array}{l}\text { Não (ref.) } \\
\text { Sim }\end{array}$ & 0,020 & $\begin{array}{c}1 \\
2,9\end{array}$ & $1,2-7,2$ \\
\hline $\begin{array}{l}\text { Discriminação contra } \\
\text { aparência física }\end{array}$ & $\begin{array}{l}\text { Não (ref.) } \\
\text { Sim } \\
\end{array}$ & 0,039 & $\begin{array}{c}1 \\
1,9 \\
\end{array}$ & $\begin{array}{c}-- \\
1,04-3,6 \\
\end{array}$ \\
\hline
\end{tabular}




\begin{tabular}{|c|c|c|c|c|}
\hline Variáveis selecionadas* & Categorias & P-Valor & $\mathbf{O R}^{* *}$ & IC 95\% OR \\
\hline \multicolumn{5}{|c|}{ Sintomas persistentes de ansiedade mais que o habitual } \\
\hline Sexo & $\begin{array}{l}\text { Masc.(ref.) } \\
\text { Fem. }\end{array}$ & 0,001 & $\begin{array}{c}1 \\
2,3\end{array}$ & $1,4-3,7$ \\
\hline $\begin{array}{l}\text { Discriminação contra } \\
\text { desempenho acadêmico }\end{array}$ & $\begin{array}{l}\text { Não (ref.) } \\
\text { Sim }\end{array}$ & 0,007 & $\begin{array}{c}1 \\
3,3 \\
\end{array}$ & $\begin{array}{l}--- \\
1,4-8\end{array}$ \\
\hline Idade & $\begin{array}{c}\leq 20 \text { (ref.) } \\
21-25 \\
\geq 26\end{array}$ & $\begin{array}{l}0,020 \\
0,025\end{array}$ & $\begin{array}{c}1 \\
1,9 \\
2,0\end{array}$ & $\begin{array}{c}--- \\
1,1-3,3 \\
1,1-3,8\end{array}$ \\
\hline \multicolumn{5}{|c|}{ Insônia por pelo menos 2 semanas nos últimos 12 meses } \\
\hline $\begin{array}{l}\text { Discriminação contra cor } \\
\text { da pele }\end{array}$ & $\begin{array}{l}\text { Não (ref.) } \\
\text { Sim }\end{array}$ & $<0,001$ & $\begin{array}{c}1 \\
5,6\end{array}$ & $2,2^{---}-13,8$ \\
\hline $\begin{array}{l}\text { Discriminação contra } \\
\text { status social }\end{array}$ & $\begin{array}{l}\text { Não (ref.) } \\
\text { Sim }\end{array}$ & 0,005 & $\begin{array}{c}1 \\
2,1\end{array}$ & $1,2-3,5$ \\
\hline \multicolumn{5}{|c|}{ Pensamentos suicidas pelo menos uma vez na vida } \\
\hline $\begin{array}{l}\text { Discriminação contra } \\
\text { aparência física }\end{array}$ & $\begin{array}{l}\text { Não (ref.) } \\
\text { Sim }\end{array}$ & $<0,001$ & $\begin{array}{c}1 \\
3,1\end{array}$ & $1,7-5,8$ \\
\hline
\end{tabular}

Fonte: Pesquisa de Campo (DOS SANTOS-JÚNIOR, 2011)

* Ref: nível de referência, critério Stepwise para seleção de variáveis

** $\mathrm{OR}=$ Odds Ratio para M.I.N.I. positivo; CI 95\% OR= Intervalo de confiança de $95 \%$ para OR

$\S$ negativo $(\mathrm{n}=128)$; positivo $(\mathrm{n}=196) / \S \S$ negativo $(\mathrm{n}=253)$; positivo $(\mathrm{n}=70) / \S \S \S$ negativo $(\mathrm{n}=249)$; positivo $(\mathrm{n}=74) /+$ negativo $(\mathrm{n}=272)$; positivo $(\mathrm{n}=50) /++$ negativo $(\mathrm{n}=268)$; positivo $(\mathrm{n}=54) /+++$ negativo $(\mathrm{n}=185)$; positivo $(\mathrm{n}=137)$ / \# negativo $(\mathrm{n}=207)$; positivo $(\mathrm{n}=113)$ / \#\# negativo $(\mathrm{n}=272)$; positivo $(\mathrm{n}=48)$

${ }^{* * *}$ Sem significância estatística $(\mathrm{p}>0,05)$ na comparação entre os subgrupos "21-25 anos e menores de 21 anos"

Outros efeitos comportamentais, associados com o uso mais frequente de álcool e de substâncias psicoativas ilícitas, bem como os comportamentos de risco após usálas, apresentaram associações estatisticamente significativas com as variáveis independentes, como mostra a Tabela 4. 
Tabela 4 - Análises de regressão logística multivariada para resultados associados ao uso de substâncias psicoativas

\begin{tabular}{|c|c|c|c|c|}
\hline Variáveis selecionadas* & Categorias & P-Valor & OR** & IC 95\% OR \\
\hline \multicolumn{5}{|c|}{ Uso mais frequente de álcool } \\
\hline Trabalho & $\begin{array}{l}\text { Sim (ref.) } \\
\text { Não }\end{array}$ & 0,005 & $\begin{array}{c}1 \\
2,6\end{array}$ & $1,3-5,1$ \\
\hline $\begin{array}{l}\text { Discriminação contra } \\
\text { roupas e adereços corporais }\end{array}$ & $\begin{array}{l}\text { Sim (ref.) } \\
\text { Não }\end{array}$ & 0,008 & $\begin{array}{c}1 \\
2,7\end{array}$ & $1,3-5,5$ \\
\hline Idade & $\begin{array}{c}\geq 26 \text { (ref.) } \\
\leq 20 \\
21-25\end{array}$ & $\begin{array}{c}0,350^{* * *} \\
0,017\end{array}$ & $\begin{array}{c}1 \\
1,6 \\
3,1\end{array}$ & $\begin{array}{c}--- \\
0,6-4,6 \\
1,2-7,7\end{array}$ \\
\hline $\begin{array}{l}\text { Discriminação contra } \\
\text { religião }\end{array}$ & $\begin{array}{l}\text { Sim (ref.) } \\
\text { Não }\end{array}$ & 0,030 & $\begin{array}{c}1 \\
4,2\end{array}$ & $1,2-15,4$ \\
\hline \multicolumn{5}{|c|}{ Uso de substâncias psicoativas ilegais } \\
\hline $\begin{array}{l}\text { Discriminação contra } \\
\text { posições políticas }\end{array}$ & $\begin{array}{l}\text { Sim (ref.) } \\
\text { Não }\end{array}$ & 0,031 & $\begin{array}{c}1 \\
2,5\end{array}$ & $1,1-5,6$ \\
\hline \multicolumn{5}{|c|}{ Comportamento de risco após o uso de álcool ou substâncias psicoativas ilegais } \\
\hline Sexo & $\begin{array}{l}\text { Masc. (ref.) } \\
\text { Fem. }\end{array}$ & 0,006 & $\begin{array}{c}1 \\
2,3 \\
\end{array}$ & $1,3-4,2$ \\
\hline $\begin{array}{l}\text { Período que frequenta a } \\
\text { faculdade }\end{array}$ & $\begin{array}{c}\text { Noturno } \\
\text { (ref.) } \\
\text { Diurno }\end{array}$ & 0,019 & $\begin{array}{c}1 \\
2,3\end{array}$ & $1,1-4,5$ \\
\hline $\begin{array}{l}\text { Discriminação } \\
\text { contra cor da pele }\end{array}$ & $\begin{array}{l}\text { Não (ref.) } \\
\text { Sim }\end{array}$ & 0,025 & $\begin{array}{c}1 \\
2,8\end{array}$ & $1,1-6,8$ \\
\hline
\end{tabular}

Fonte: Pesquisa de Campo (DOS SANTOS-JÚNIOR, 2011)

"Ref: nível de referência, critério Stepwise para seleção de variáveis

${ }^{* *} \mathrm{OR}=$ Odds Ratio para M.I.N.I. positivo; CI 95\% OR= Intervalo de confiança de $95 \%$ para OR.

*** Sem significância estatística $(p>0,05)$ na comparação entre os subgrupos "menores de 21 anos e maiores de 25 anos"

$\S$ negativo $(\mathrm{n}=254)$; positivo $(\mathrm{n}=66)$ / $\S$ negativo $(\mathrm{n}=241)$; positivo $(\mathrm{n}=78) / \S \S \S$ negativo $(\mathrm{n}=247) ;$ positivo $(n=64)$

\section{DISCUSSÃO}

- A composição da amostra

Os primeiros passos do processo de composição da amostragem deste estudo estiveram de acordo com os resultados das pesquisas realizadas em outras universidades públicas brasileiras, em que se constatava a sub-representação de estudantes pardos e negros na graduação, como em trabalho realizado por Queiroz (2004). Enquanto, segundo o IBGE, indivíduos pardos e negros, juntos, compõem 46\% da população Serv. Soc. \& Saúde, Campinas, SP v. 14, n. 2 (20), p. 273-298, jul./dez. 2015 ISSN 1676-6806 
brasileira, neste inquérito eram responsáveis por apenas $14 \%$ da soma dos estudantes que primeiro responderam ao questionário. O grande tamanho da amostra inicial permitiu considerar, para a construção de amostragem final, dois grupos, de acordo com a cor de pele: ou composto de estudantes autodeclarados brancos e outro de estudantes autodeclarados negros ou pardos, tornando também possível o procedimento de emparelhamento estratificado, o que é inédito no sentido de permitir que a expressão de características e pontos de vista de minorias raciais e socioeconômicas dos estudantes de graduação brasileiros.

- Queixas e sintomas

Neste estudo, o nível socioeconômico não apresentou diferença estatística em relação a piores resultados em saúde mental nas categorias examinadas. Alunos negros e pardos apresentaram maiores taxas de episódios depressivos maiores ${ }^{6}$ e relataram significativamente mais experiências de discriminação com base na cor da pele e desempenho acadêmico. Após o procedimento de regressão logística multivariada isso não foi refletido na associação com piores resultados de saúde mental nas principais categorias testadas. Este resultado não seria evidente se a amostra não fosse previamente estratificada, de acordo com características socioeconômicas, entre os diferentes grupos de cor de pele. É importante ter em conta que os indivíduos negros e pardos, além de serem sub-representados no cenário do ensino superior público brasileiro, são também, em sua maioria, provenientes de famílias de baixo nível socioeconômico. Apenas itens que não se configuram em possíveis diagnósticos específicos tiveram associação com discriminação contra a cor de pele. A associação encontrada com insônia e comportamento de risco após uso de álcool ou substâncias psicoativas ilícitas, entretanto, é uma motivação para que particularidades deste grupo sejam consideradas em pesquisas futuras, para melhor compreensão deste padrão de comportamento social.

Houve associação entre ser do sexo feminino e queixas indicativas de mais sofrimento psicológico internalizado (qualquer categoria afirmativa avaliada pelo M.I.N.I.), principalmente quadros sugestivos de transtorno de ansiedade generalizada e distimia. Resultados semelhantes de relatos mais frequentes de estresse psicológico

\footnotetext{
${ }^{6}$ Utilizamos o termo "episódio depressivo maior", que engloba depressão de moderada e grave intensidade.
} 
entre as mulheres na mesma população da Universidade foram encontrados, como observado por Neves (2007). Em contraste, os estudantes do sexo masculino foram mais associados com mais relatos de comportamentos de risco.

Associações de idade com variáveis dependentes foram diferentes para as faixas etárias estudadas. Houve associação entre pertencer ao grupo entre 21 e 25 anos e sintomas atuais auto avaliados de condições de internalizadas, como sintomas depressivos e ansiosos. Além das categorias avaliadas pelo M.I.N.I., os estudantes dessa faixa etária também foram aqueles com uso mais frequente de álcool, enfatizando que pode haver um grande risco para o abuso de álcool e suas consequências neste período do desenvolvimento. O final da adolescência e o início da idade adulta constituem períodos de grandes mudanças e que permitem aos estudantes engendrarem grandes oportunidades de amadurecimento. São, todavia, períodos também usualmente acompanhados de bastante estresse psicológico, podendo precipitar a ocorrência ou recorrência de transtornos mentais.

Os alunos que não trabalhavam tiveram uma tendência ao uso mais frequente de álcool e aqueles que frequentavam as aulas durante o dia reportaram mais comportamentos de risco depois de beber ou usar substâncias psicoativas. Estes estudantes universitários tenderam a ser mais jovens e de maior nível socioeconômico. Eles costumavam realizar mais atividades de lazer com os seus amigos, como festas, em que o uso de álcool ou outras substâncias psicoativas não são incomuns. Jovens adultos que usam álcool e drogas também podem estar em risco para outros transtornos psiquiátricos e para uma menor realização educacional, o que é ressaltado nos resultados obtidos por King (2006) e Moore (2007), mostrando que estes adultos jovens podem ter menor sucesso profissional.

- Tipos de discriminação auto percebida

Com a exceção da orientação sexual, que não mostrou associação estatisticamente significativa com qualquer conjunto de sintomas ou queixas, possivelmente devido ao pequeno número de indivíduos dessa amostra que relataram ter sofrido este tipo de discriminação, todas as outras variáveis foram associadas com algum efeito na saúde mental, como um cluster indicado pelo instrumento M.I.N.I., ou como um marcador do uso de substâncias psicoativas, incluindo álcool.

Serv. Soc. \& Saúde, Campinas, SP v. 14, n. 2 (20), p. 273-298, jul./dez. 2015 ISSN 1676-6806 
Além de ser objetivamente alvo de discriminação ou subjetivamente se sentindo assim, vivências de discriminação percebidas foram relatadas pela maioria dos estudantes $(77,5 \%)$ e foram distribuídas ao longo de categorias distintas. De acordo com os pressupostos dos autores, essas categorias podem representar duas concepções diferentes de perceber-se discriminados, que são separados aqui como pessoas que se sentem "inferiores" e aqueles que se sentem "diferentes".

\section{A) Os que podem se sentir "inferiores"}

A primeira concepção pode incorporar sentimentos de inferioridade, que podem ser explicados pelos significados análogos semânticos das palavras que indicam baixa autoestima, como "feio", "pobre", "burro" ou "perdedor". Discriminação contra a aparência física, status socioeconômico e desempenho acadêmico são exemplos típicos e as duas primeiras foram as categorias mais citadas de discriminação por aqueles que sentiram qualquer uma delas, bem como aquelas relacionadas a mais queixas psicológicas internalizadas.

B) Aqueles que podem ter se sentido "o único desse tipo"

A segunda concepção quanto ao modo de se sentir discriminado não é exatamente associada com experiências de inferioridade, mas, em vez disso, pode ser pensada como o indivíduo se identificar como sendo membro de um grupo específico, minoritário. Trata-se de indivíduos que se veem como pessoas fora do mainstream ${ }^{7}$, principalmente por causa de escolhas pessoais, credos ou traços raciais. Nesta categoria, ao invés de baixa autoestima, os significados predominantes de percepções são aqueles relacionados com palavras como "estranho" ou "esquisito". Indivíduos podem se sentir diferentes dos demais por variadas razões, o que também se reflete em variados tipos de associações com sintomas psicológicos e comportamentais, quando sentem que são discriminados devido a essas razões diversificadas. Categorias desta concepção incluem, predominantemente, a discriminação contra a cor da pele, formas particulares de se vestir e usar adornos corporais, ser membro de determinada religião ou defender pontos de vista políticos ou ideológicos específicos e minoritários. Nenhuma dessas

\footnotetext{
${ }^{7} \mathrm{O}$ termo "mainstream" é um conceito que expressa uma tendência ou moda dominante. A tradução literal de mainstream é "corrente principal" ou "fluxo principal".

Serv. Soc. \& Saúde, Campinas, SP v.15, n. 2 (22), p. 273-298, jul./dez. 2016 ISSN 1676-6806
} 
categorias foi, no presente estudo, associada com condições depressivas, mas sim com queixas de ansiedade e diferentes padrões de condições externalizantes, como o uso de substâncias psicoativas, incluindo o álcool.

Os estudantes que relataram a discriminação com base na cor da pele foram aqueles com maior comportamento de risco depois de beber ou usar substâncias psicoativas. Aqueles que declararam experiências de discriminação devido a roupas e adereços corporais apresentaram níveis mais elevados de problemas relacionados ao álcool, o oposto do que foi encontrado no caso daqueles que relataram experiências de discriminação com base na religião, provavelmente porque as tradições cristãs evangélicas e católicas no Brasil desaprovam o uso indevido de álcool. Discriminação relatada contra posições políticas mostrou-se associada com taxas mais altas de uso de substâncias psicoativas ilícitas. Uma possível ligação é que as pessoas que reportaram sofrer discriminação por motivos políticos tenderam a ser aquelas que assumiram posições explicitamente mais contrárias a regras e regulamentos. O uso de substâncias psicoativas ilícitas pode representar, nesse contexto, um importante elemento de protesto.

\section{LIMITAÇÕES}

A análise deste estudo é derivada de dados secundários, o que não permitiu a investigação de outras variáveis de possível interesse. No entanto, foi possível analisar informações importantes, recolhidas a partir das respostas a um inquérito amplo e detalhado.

A estratégia de dividir os alunos de graduação em dois grupos, de acordo com a cor de pele, e emparelhá-los de acordo com medidas indiretas de nível socioeconômico dos alunos negros e pardos implica o risco de um possível viés de seleção, em direção à hipótese de nenhuma associação em variáveis relacionadas ao nível socioeconômico. Isso pode dificultar a análise estatística, por não equilibrar as variáveis de possível confusão. A generalização dos resultados, no entanto, não foi prejudicada porque os alunos foram selecionados a partir da mesma população e, também, porque as variáveis controladas foram aquelas relacionadas com as diferenças encontradas entre os grupos antes do procedimento de emparelhamento.

Serv. Soc. \& Saúde, Campinas, SP v. 14, n. 2 (20), p. 273-298, jul./dez. 2015 ISSN 1676-6806 


\section{CONCLUSÃO}

Queixas psicológicas, uso de substâncias psicoativas e consequentes comportamentos de risco foram frequentes entre o grupo de estudantes de graduação brasileiros investigados neste estudo, assim como percepções de diferentes categorias de experiências discriminatórias. Tipos distintos de discriminação foram associados com dois principais padrões de resultados: aqueles que produzem sentimentos de inferioridade (discriminação contra aparência física, status socioeconômico e desempenho acadêmico), sendo mais frequentemente associados com queixas internalizadas; e aqueles que contêm a concepção de ser diferente da maioria (discriminação contra as posições políticas, roupas e adornos corporais, religião, cor da pele), mais frequentemente associados com a ansiedade $\mathrm{e}$ atitudes de risco externalizantes. Estudos futuros deverão explorar o impacto longitudinal de cada categoria de discriminação percebida e associações causais com diagnósticos psiquiátricos específicos, consequências adversas pessoais e sociais e a busca de ajuda ou tratamento.

\section{AGRADECIMENTO}

À Mestre Marly Coelho Carvalho Neves, psicóloga. Ela é a admirável idealizadora, aplicadora dos questionários e organizadora do banco de dados, de onde foram obtidas as informações que permitiram a efetivação deste estudo.

Recebido em 22.06.2016 - Aprovado em 29.12.2016

\section{REFERÊNCIAS}

KELLY, B. D.; FEENEY, L. Textbook of cultural psychiatry. Cambridge, UK:

Cambridge University Press, p. 550-560, 2007.

BHUGRA, D.; AYONRINDE, O. Racism, racial life events and mental ill health.

Advanced Psychiatry Treat, v. 7, p. 216-23, 2001. 
NOH, S.; KASPAR, V.; WICKRAMA; K. A. S. Overt and subtle racial discrimination and mental health: Preliminary findings for Korean immigrants. American Journal of Public Health, v. 97, p. 1269-74, 2007.

ELLIS, B. H.; MACDONALD, H. Z.; LINCOLN, A. K.; CABRAL, H. J. Mental health of Somali adolescent refugees: The role of trauma, stress, and perceived discrimination. Journal Consult of Clinical Psychology, v. 76, p. 184-93, 2008.

LANDRINE, H.; KLONOFF, E. A.; CORRAL, I.; FERNANDEZ, S., ROESCH, S. Conceptualizing and measuring ethnic discrimination in health research, Journal of Behaviour Medicine, v. 29, p. 79-94, 2006.

HWANG, W. C.; GOTO, S. The impact of perceived racial discrimination on the Mental health of Asian American and Latino college students. Cultural Diversity of Ethnics Minor Psychology, v. 14, p. 326-35, 2008.

OLIVEIRA, M. L. C.; DANTAS, C. R.; AZEVEDO, R. C. S.; BANZATO, C. E.M. Counseling Brazilian undergraduate students: 17 years of a campus mental health service. Journal of American College Health, v. 57, p. 367-72, 2008.

PRELOW, H.M.; MOSHER, C. E.; BOWMAN, M. A. Perceived racial discrimination, social support, and psychological adjustment among African American college students. Journal of Black Psychology, v. 32, p. 442-54, 2006.

LAHIRI, K.; RETTIG-EWEN, V.; BOHM, M.; LAUFS, U. Perceived psychosocial stress and cardiovascular risk factors in obese and non-obese patients. Clinical Resident of Cardiology, v. 96, p. 365-74, 2007.

LO, W. S.; HO, S. Y.; MAK, K.; WONG, Y.; LAI, Y.; LAM, T. Prospective effects of weight perception and weight comments on psychological health among Chinese Adolescents. Acta Paediatric, v. 98, p. 1959-64, 2009.

GRUNBAUM, J.; KANN, L.; KITCHEN, S.; ROSS, J.; HAWKINS, J.; LOWRY, R. et al. Youth Risk Behavior Surveillance, United States MMWR. Morbidity and Mortality Weekly Report, v. 53, p. 1-96, 2004.

VINER, R. M. ; HAINES, M. M.; TAYLOR, S. J. C.; HEAD, J.; BOOY, R.; STANSFELD, S. Body Mass, weight control behaviors, weight perception and emotional wellbeing in a multiethnic sample of early adolescents. Internal Journal of Obessity, v. 30, p. 1514-21, 2006. 
THE INTERNATIONAL ASSOCIATION OF COUNSELING SERVICES. National Survey of Counseling Center Directors, 2009. Disponível em:

$<$ http://www,iacsinc,org/2009\%20National\%20Survey,pdf $>$, Acesso em 06 ago, 2015, SOMERS, J. M.; GOLDNER, E. M.; WARAICH, P.; HU, L. Prevalence studies of substance-related disorders: A systematic review of the literature. Canadian Journal of Psychiatry, v. 49, p. 373-84, 2004.

BARRETT, S. P.; DARREDEAU, C.; PIHL, R. O. Patterns of simultaneous polysubstance use in drug using University students. Human Psychopharmacology, v. 21, p. 255-63, 2006.

HINGSON, R.; HEEREN, T.; WINTER, M.; WECHSLER, H. Magnitude of alcoholrelated mortality and morbidity among U.S. college students aged 18-24: Changes from 1998 to 2001. Annual Revision of Public Health, v. 26, p. 259-79, 2005.

BLANCO, C.; OKUDA, M. WRIGHT, B. S.; HASIN, .S.; GRANT, B. F.; LIU, S. et al. Mental health of college students and their non-college-attending peers: Results from the National Epidemiologic Study on Alcohol and Related Conditions. Archieve of General Psychiatry, v. 65, p. 1429-37, 2008.

MCCORMACK, A. S. Revisiting discrimination on campus: 1988, 1992, 1996, College Students Journal, v. 32, p. 378-93, 1996.

UNICAMP. A Universidade Estadual de Campinas em números. Anuário Estatístico, 2016. Disponível em:

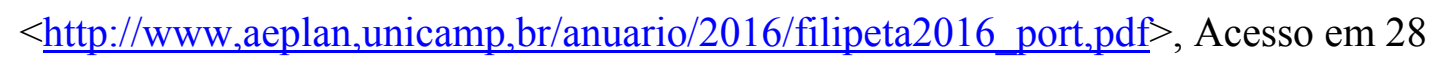
dez. 2016.

NEVES, M. C. C.; DALGALARRONDO, P. Transtornos mentais auto-referidos em Estudantes Universitários. Jornal Brasileiro de Psiquiatria. V. 56, p. 237-44, 2007. IBGE - Instituto Brasileiro de Geografia e Estatística. Censo Demográfico. 2000. Disponível em:

$<\underline{\text { http://www,ibge,gov,br/english/estatistica/populacao/censo2000/tabelabrasil131,shtm> }}$ Acesso em 13 ago. 2015.

HERINGER, R. Desigualdades raciais no Brasil: Síntese de indicadores e desafios no campo das políticas públicas. Caderno de Saúde Pública, v. 18, p. 57-65, 2002.

CHOR, D.; LIMA, C. R. A. Aspectos epidemiológicos das desigualdades raciais em saúde no Brasil. Caderno de Saúde Pública, v. 21, p. 1586-94, 2005. 
CAREY, T. S.; HOWARD, D. L. The development of the field of health disparities research: The role of cross-disciplinary and institutional collaboration, HHPR Harvard Health Policy Research, v. 8, p. 136-44, 2007.

SHEEHAN, D.; LECRUBIER, Y.; SHEEHAN, K. H.; JANAVS, J.; WEILLER, E.; KESKINER, A., et al. The validity of the Mini International Neuropsychiatric Interview (M.I.N.I.) according to the SCID-P and its reliability. Euroupean Psychiatry, v. 12, p. 232-41, 1997.

AMORIM, P, Mini International Neuropsychiatric Interview (M.I.N.I.): Validação de entrevista breve para diagnóstico de transtornos mentais. Revista Brasileira de Psiquiatria, v. 22, p. 106-15, 2000.

BABOR, T. F.; FUENTE, J. R.; SAUNDERS, J.; GRANT, M. AUDIT - The Alcohol Use Disorders Identification Test: Guidelines for use in primary health care, Geneva, Switzerland: WHO (World Health Organization), 1992. LIMA, C.; FREIRE, A. C. C.; SILVA, A. P. B.; TEIXEIRA, R. M.; FARRELL, M.; PRINCE, M. Concurrent and construct validity of the Audit in an urban Brazilian sample. Alcohol and Alcoholism, v. 40, p. 584-89, 2005.

ABDEL-KHALEK, A.; LESTER, D. Can personality predict suicidality? A study in two cultures. International Journal of Social Psychiatry, v. 48, p. 231-9, 2002. DOS SANTOS-JÚNIOR, A. Identidade, discriminação e saúde mental em estudantes universitários. 2011. 304 f. Dissertação (Mestrado em Ciências, área de concentração em Saúde da Criança e do Adolescente), Universidade Estadual de Campinas, Unicamp, 2011. Disponível em $<$ http://www.bibliotecadigital.unicamp.br/document/?code $=000799132 \& \mathrm{fd}=\mathrm{y}>$, Acesso em 28 dez, 2016.

QUEIROZ, D. M. O Negro e a Universidade brasileira, Historia Actual on line, v. 3, p.

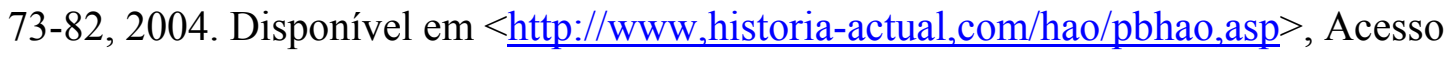
em 03 abr. 2011.

RENSSELAER POLYTECHNIC INSTITUTE. Black students / white campus: The pervasiveness of racism, 2000. Disponível em:

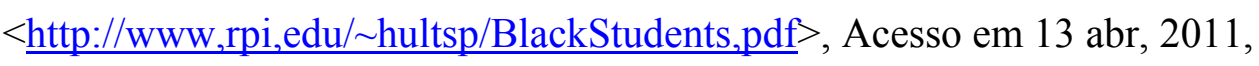


KING, K. M.; MEEHAN, B. T.; TRIM, R. S.; CHASSIN, L. Marker or mediator? The effects of substance use on young adult educational attainment. Addiction, v. 101, p. 1730-40, 2006.

MOORE, T. H. M.; ZAMMIT, S.; LINGFORD-HUGHES, A.; BARNES, T. R. E.; JONES, P. B. ; BURKE, M.; et al. Cannabis use and risk of psychotic or affective mental health outcomes: A systematic review. Lancet, v. 370, p. 319-28, 2007. 
